\title{
ON THE DENSITY OF THE INVERTIBLE GROUP IN $C^{*}$-ALGEBRAS
}

\author{
by GUYAN ROBERTSON
}

(Received 9th January 1975)

1.

In what follows the term $C^{*}$-algebra will mean a complex $C^{*}$-algebra with identity. We denote the identity element by 1 . We shall also use the notation and terminology of Dixmier (3) without comment.

Let $A$ be a $C^{*}$-algebra. It is well known that the group $G(A)$ of invertible elements of $A$ is "large", in the sense that its subgroup $U(A)$ of unitary elements actually spans the algebra. Our first result shows that $G(A)$ is in fact always dense in $A$ in the weak (Banach space) topology. The situation is more complicated when we look at the norm topology on $A$. For example, if $M$ is a von Neumann algebra then Choda (2) has shown that $M$ has dense invertible group if and only if $M$ is finite. Now suppose $C$ is an abelian $C^{*}$ algebra, with maximal ideal space $X$. It is easily seen from (8, Theorem VII 4) that $C$ has dense invertible group if and only if the topological covering dimension of $X$ is less than 2. We use this to give a necessary condition for a homogeneous $C^{*}$-algebra to have dense invertible group.

We conclude in Section 4 with some miscellaneous results and remarks.

\section{2.}

Our result for the weak topology is based on a Proposition of Dixmier and Maréchal (4), which states that in a von Neumann algebra the invertible group is always dense in the strong operator topology.

Proposition 1. Let $A$ be a $C^{*}$-algebra. Then $G(A)$ is weakly dense in $A$.

Proof. Let $A$ act on the Hilbert space $H$ in its universal representation. Then the strong operator closure $A^{-}$of $A$ is the enveloping von Neumann algebra of $A$.

Let $a$ be an element of the closed unit ball $A_{1}$ of $A$, and let $V$ be a strong neighbourhood of $a$ in $A^{-}$. By (4), $G\left(A^{-}\right)$is strongly dense in $A^{-}$. Hence there exists an element $x$ in $V \cap G\left(A^{-}\right)$. Now $x$ has polar decomposition $x=v h$, where $v$ is a unitary in $A^{-}$, and $h$ is a positive element in $A_{1}^{-}$. By the Kaplansky density theorem, the positive part of $A_{1}$ is strongly dense in the positive part of $A_{1}^{-}$. By the Glimm-Kadison density theorem (6, Theorem 2), $U(A)$ is strongly dense in $U\left(A^{-}\right)$. Also multiplication is a strongly continuous map on $U\left(A^{-}\right) \times\left(A^{-}\right)_{+}$. Hence there exists $u \in U(A)$ and $h^{\prime} \in A_{+}$such that 
$u h^{\prime} \in V$. By the functional calculus $(3,1.5), h^{\prime}$ may be approximated in norm by a positive invertible element $k \in A$, so that $u k \in V$. Now $u k \in G(A)$. Hence $G(A)$ is strong operator dense in $A$. Hence $G(A)$ is ultraweakly dense in $A$. However, since $A$ is acting in its universal representation, the ultraweak topology, when restricted to $A$, coincides with the weak (Banach space) topology of $A$. This proves the result.

It is very easy to give an example of a general Banach algebra for which the above conclusion does not hold. For let $B$ be the disc algebra. Then $B$ consists of those continuous complex valued functions on the space $\Sigma$ of complex numbers of modulus $\leqq 1$, which are analytic in the interior of $\Sigma$. Let $f \in B$ have a zero at some point in the interior of $\Sigma$, and suppose that $f$ is not identically zero. We claim that $f$ does not lie in the weak closure of $G(B)$.

For suppose $\left(f_{\gamma}\right)$ is a net in $G(B)$ which converges weakly to $f$. By the principle of uniform boundedness the set $\left(\left\|f_{\gamma}\right\|\right)$ is bounded. Also $f_{\gamma} \rightarrow f$ pointwise on $\Sigma$. By $\left(1\right.$, p. 171, Theorem 9) the family $\left(f_{v}\right)$ is normal. Hence, by $\left(1, \mathrm{p} .171\right.$, Corollary), since each $f_{v}$ is never zero, a limit function of the set $\left(f_{y}\right)$ is either never zero or is identically zero. But this contradicts our choice of $f$, and hence our claim is proved.

We note that in the above example the norm and the weak closures of $G(B)$ in fact coincide. For if a function $f$ in $B$ is either identically zero or has all its zeros contained in the boundary of $\Sigma$ then it clearly lies in the norm closure of $G(B)$.

We turn now to the more difficult question of the norm density of the invertible group in $C^{*}$-algebras. We noted earlier that the answer is known completely in the case of abelian $C^{*}$-algebras. Now the simplest class of non-commutative $C^{*}$-algebras is that of homogeneous $C^{*}$-algebras. Recall that a $C^{*}$-algebra $A$ is called homogeneous of degree $n$ ( $n$ a positive integer) if all its irreducible representations are of degree $n$. We are able to provide an answer to our question in the case of some such algebras.

Let $A$ be an $n$-homogeneous $C^{*}$-algebra (for some positive integer $n$ ). We recall some notation from $(3,3.5)$. Let $\operatorname{Irr}_{n}(A)$ denote the set of irreducible representations of $A$ on an $n$-dimensional Hilbert space $H_{n}$. Equip $\operatorname{Irr}_{n}(A)$ with the topology of simple strong convergence on $A$. i.e. $\pi_{\gamma} \rightarrow \pi$ in $\operatorname{Irr}_{n}(A)$ means $\pi_{y}(a) \beta \rightarrow \pi(a) \beta$ for all $a \in A, \beta \in H_{n}$. $\quad \hat{A}$ denotes the set of unitary equivalence classes of irreducible representations of $A$, with the Jacobson topology. We have a canonical map $\operatorname{Irr}_{n}(A) \rightarrow \hat{A}$, which is a quotient map for the respective topologies $(3,3.5 .8)$. Let det denote the determinant on the matrix algebra $L\left(H_{n}\right)$ of all operators on $H_{n}$. Given $x \in A$, the map $\pi \rightarrow \pi(x)$ is continuous on $\operatorname{Irr}_{n}(A)$. Hence so also is the map $\pi \rightarrow \operatorname{det} \pi(x)$. This map is also constant on unitary equivalence classes of elements of $\operatorname{Irr}_{n}(A)$, and so, by passing to the quotient, defines a continuous complex-valued function $\partial_{x}$ on $\widehat{A}$.

For completeness we give the definition of covering dimension for topological spaces. 
Definition (8, p. 9). Let $X$ be a normal topological space. We say that $X$ has dimension $\leqq n$ ( $n$ a positive integer) if every finite open covering of $X$ has a finite open refinement of order $\leqq n+1$ (i.e. each point of $X$ is contained in at most $n+1$ sets of this refinement). We write this as $\operatorname{dim} X \leqq n$.

We can now prove

Proposition 2. Let $A$ be a $C^{*}$-algebra which is homogeneous of finite degree and has uniformly dense invertible group. Then $\operatorname{dim} \hat{A} \leqq 1$.

Proof. Note firstly that we are able to apply the dimension theory of (8) to $\hat{A}$ because $\hat{A}$ is a compact Hausdorff space $(3,3.1 .8$ and 3.6.4). By (8, Theorem VII 4), it suffices to show that the invertible elements are dense in $C(\hat{A})$ (the sup norm algebra of continuous complex-valued functions on $\widehat{A}$ ).

Let $f \in C(\widehat{A})$, and let $\varepsilon>0$. By a special case of the Dauns-Hofmann theorem $(3,10.5 .6)$, there exists $a \in A$ such that for all $\pi \in \hat{A}$,

$$
\pi(a)=f(\pi) \pi(1) \text {. }
$$

Now, by continuity of det and $(3,1.3 .7)$, there exists $\delta>0$ such that, if $x \in A$ and $\|x-a\|<\delta$ then

$$
|\operatorname{det} \pi(x)-\operatorname{det} \pi(a)|<\varepsilon \quad\left(\pi \in \operatorname{Irr}_{n}(A)\right)
$$

Since $G(A)$ is dense in $A$, there exists $x \in G(A)$ with $\|x-a\|<\delta$. Then, for each $\pi \in \hat{A}$,

$$
\left|\partial_{x}(\pi)-f(\pi)\right|=|\operatorname{det} \pi(x)-\operatorname{det} \pi(a)|<\varepsilon .
$$

Since $x$ is invertible, $\pi(x)$ is invertible for each $\pi \in \widehat{A}$, and so $\partial_{x}$ is non-zero on $\hat{A}$. Also, as we have already remarked, $\partial_{x} \in C(\hat{A})$. This completes the proof.

Examples of homogeneous $C^{*}$-algebras are those of the form

$$
A=C(X) \otimes M_{n},
$$

where $X$ is a compact Hausdorff space and $M_{n}$ is the full $n \times n$ matrix algebra. A routine, but tedious, argument, using induction on $n$, shows that the converse of Proposition 2 holds for such algebras. A more general result is proved in (11), using the structure theory of (5). However, we do not know whether the full converse of Proposition 2 is true or not.

4.

We now give a useful characterisation of invertible elements in $C^{*}$-algebras which have dense invertible groups.

Proposition 5. Let $A$ be a $C^{*}$-algebra with $G(A)$ dense in $A$. Let $x \in A$. The following are equivalent:
(1) $x \in G(A)$;
(2) $f\left(x^{*} x\right)>0$ for each $f \in P(A)$;
(3) $f\left(x^{*} x\right)>0$ for each $f \in E(A)$;
(4) $\pi(x)$ is invertible for each $\pi \in \hat{A}$. 
Proof. Obviously (1) implies (4) and (3) implies (2). Also, for any $C^{*}$ algebra $A$, (1) implies (3). For if $x \in G(A)$, then by $(3,2.1 .2)$, for each $f \in E(A)$,

$$
1=\left|f\left(x^{-1} x\right)\right|<f\left(x^{*} x\right) f\left(x^{-1} x^{-1 *}\right) \text {. }
$$

(2) implies (1): Suppose $x$ is a singular element of $A$. Then $x$ lies in the boundary of $G(A)$ and hence is a two-sided topological divisor of zero. Hence $x$ is not left invertible, and so $A x$ is a proper closed left ideal of $A$ containing $x$. Hence $x$ is contained in a maximal left ideal $L$ of $A$. By $(3,2.9 .5), L$ is the left kernel of some pure state $f$ of $A$, and so $f\left(x^{*} x\right)=0$.

(4) implies (1): Suppose $x$ is a singular element of $A$, and let $L$ be as above. Then the canonical representation $\pi$ of $A$ on the Hilbert space $A / L$ is irreducible and $\pi(x)$ is not invertible, since $x \in L$.

As an example of the way in which the above result may be applied we have the following

Corollary 6. Let $A$ be a liminal $C^{*}$-algebra (with identity) and let $x \in A$. Then conditions (1) to (4) of Proposition 5 are equivalent.

Proof. By Proposition 5, we need only imbed $A$ in a $C^{*}$-algebra $B$ with dense invertible group, such that every pure state of $B$ restricts to a pure state of $A$.

Let $\pi$ be the reduced atomic representation of $A$ (a direct sum of irreducible representations of $A$, taking exactly one representation from each unitary equivalence class). Then $\pi$ is a faithful representation of $A$ and the strong closure $B$ of $\pi(A)$ is a finite von Neumann algebra, and hence has dense invertible group (2, Theorem 5). It is easy to see that each pure state of $B$ restricts to a pure state of $A$.

Finally we return to the case of abelian $C^{*}$-algebras. Let $A$ be an abelian $C^{*}$-algebra with maximal ideal space $X$. We may write $A=C(X)$. As we noted previously (10), it is shown by Peck (9) that when $A$ is separable (i.e. $X$ is metric), the following are equivalent.

(1) $A_{1}=\operatorname{co} U(A)$.

(2) $\operatorname{dim} X \leqq 1$ (i.e. $A$ has dense invertible group).

In the non-separable case the proof that (2) implies (1) in (9) goes over without change using the results in (8) corresponding to those in (7). We now give a simplification of Peck's argument, which also proves that (1) implies (2) in the non-separable case.

Suppose $A=C(X)$ satisfies (1). In order to show that $\operatorname{dim} X \leqq 1$, we need only show that for every closed subset $F$ of $X$ and continuous map $g$ from $F$ into the unit circle $S=\left\{e^{i \beta}: 0 \leqq \beta \leqq 2 \pi\right\}$ there exists a continuous extension of $g$ to the whole of $X(8$, Theorem VII, 5). Take such $g$ and $F$. By the Tietze extension theorem $g$ extends to an element $\bar{g}$ of $C(X)$ with $\|\bar{g}\| \leqq 1$. By hypothesis $\bar{g}$ can be expressed as a convex combination of 
unitaries in $C(X)$, i.e. there exist positive scalars $\beta_{k}$ and maps $u_{k}$ from $X$ into $S$ with

$$
\bar{g}=\sum_{k=1}^{n} \beta_{k} u_{k}, \quad \sum_{k=1}^{n} \beta_{k}=1 .
$$

Now for $x \in F,|g(x)|=1$ and

$$
g(x)=\bar{g}(x)=\sum_{k=1}^{n} \beta_{k} u_{k}(x) .
$$

Hence $g(x)=u_{k}(x)(1 \leqq k \leqq n)$. Thus any $u_{k}$, say $u_{1}$, will give the required extension.

\section{REFERENCES}

(1) L. Ahlfors, Complex analysis (McGraw-Hill, 1952).

(2) H. CHODA, An extremal property of the polar decomposition in von Neumann algebras, Proc. Japan Acad. 46 (1970), 341-344.

(3) J. Dixmier, Les $C^{*}$-algèbres et leurs representations (Gauthier-Villars, 1969).

(4) J. Dixmier and O. Maréchal, Vecteurs totalisateurs d'une algèbre de von Neumann, Commun. Math. Phys. 22 (1971), 44-50.

(5) J. M. G. Fell, The structure of algebras of operator fields, ${ }^{-}$Acta Math 106 (1961), 233-280.

(6) J. Glimm and R. V. Kadison, Unitary operators in $C^{*}$-algebras, Pac. J. Math. 10 (1960), 547-556.

(7) W. Hurewicz and H. Wallman, Dimension theory (Princeton University Press, 1948).

(8) J. Nagata, Modern dimension theory (North Holland, 1965).

(9) N. T. PECK, Representation of functions in $C(X)$ by means of extreme points, Proc. Amer. Math. Soc. 18 (1967), 133-135.

(10) A. G. Robertson, A note on the unit ball in $C^{*}$-algebras, Bull. London Math. Soc. 6 (1974), 333-335.

(11) A. G. Robertson, Ph.D. Thesis (Newcastle University, 1974).

Mathematical InStitute

20 Chambers STREeT

EDINBURGH EH1 $1 \mathrm{HZ}$ 